\title{
Knowledge Service Model for Business Process Design
}

\author{
Ludmila Penicina \\ Department of Systems Theory and Design \\ Riga Technical University \\ Riga, Latvia \\ ludmila.penicina@rtu.lv
}

\begin{abstract}
Business processes are key corporate assets both generating and requiring knowledge. During business process modeling phase in order to create an accurate and realistic process model a business process analyst requires existing business process knowledge embedded in internal organizational resources such as documentation and other software artifacts as well as in external resources such as legal documents repositories, standards, regulations and business process frameworks. The aim of this research is to design a service model that will identify and extract knowledge related to business processes from existing external and internal sources and store it as ontology enabling extracted knowledge to be represented in a machine readable format. This research is in the initial analysis phase of the first year of the doctoral studies.
\end{abstract}

Keywords-business process knowledge; business process ontology; information extraction.

\section{INTRODUCTION}

Nowadays regular changes imposed by external and internal factors are inevitable in every organization. To cope with steady changing environments and tackle the issues of preserving existing knowledge and producing new knowledge organizations implement knowledge management systems. Knowledge management empowers continuous learning of the organization and sustains organizational memory ensuring retention of the knowledge in constantly changing setting. In parallel with knowledge management organizations are adopting business process management approach to increase process efficiency and enhance value delivery to customers.

Business processes is a fundamental part of any organization independent of its size, industry or its market share. A process is a completely closed, time-logical sequence of activities that are required for working on a process-oriented relevant business object [1]. Organizational business processes are key corporate assets comprising vital organizational knowhow knowledge. Business process models are both generating knowledge and requiring knowledge. The complexity of modern business processes demand organizations to put into operation IT systems to support and automate their business processes. Businesses today implement Business Process Management solutions to manage improvements of business processes, to cope with growing complexity of transactions in a contemporary business setting and to provide agility to an organization as the ability of an enterprise to sense and predict change in their environment and respond quickly, efficiently, and effectively to that change [1].

During business process modeling phase a business process analyst requires existing knowledge about the process. Harvesting existing knowledge about the process is substantial while creating "as-is" process models. Usually existing business process knowledge is communicated during the interviews with subject matter experts and management, obtained from information systems and other related project documentation or gained during software reviews. Required business process knowledge is not limited to organizational boundaries and can be maintained in organization's external environment e.g. laws and regulations, standards and the best practices.

The aim of this research is to create a service model that in a standardized way will identify and retrieve existing process knowledge from internal organizational resources and external repositories e.g. legal documents repositories, process classification frameworks, etc. The service will provide an access for business analysts to the existing explicit knowledge about the process in the real-time mode. The innovative service feature is the component that will identify, retrieve and deliver the existing business process knowledge from internal organizational resource or external source evolving the notion of "business process knowledge as a service".

The novelty of the proposed approach resides in the capability of the service to harvest internal and external sources for business process knowledge, to extract business process knowledge according to the request jointly from different organizational and external resources, to integrate extracted knowledge, to create a formalized structure of the extracted knowledge in the form of the ontology and to present the ontology to the business process modeler. This goes beyond the existing state of the art through the introduction of the service extracting knowledge jointly from different sources and providing business analyst with the integrated and formalized knowledge according to the search request.

The business process modeler will be presented with the extracted knowledge using the tree structure containing business process knowledge ontology classes ordered by relevance to the query containing source name, excerpt of the requested knowledge of the business process and links to the source. The modeler using a "drag and drop" technique will be 
able to connect the extracted knowledge to a business process model element in this way specifying the source of the information related to this particular element in the business process model.

Business Process Model and Notation (BPMN) [2] is the de-facto standard for representing in a very expressive graphical way the processes occurring in virtually every kind of organization [3]. In the context of this research BPMN is used as a base notation for business process modeling. BPMN provides constructs to tackle the issue of modeling externally stored rules within a business process and reusing existing BPMN process models (see Related Work). During this research BPMN is analyzed in the context of supporting realization of business process ontology and as a result if necessary proposing an extension for BPMN meta-model to address the issue of storing and presenting business process knowledge as ontology.

\section{A. Motivation and Problem Statement}

Business process knowledge in organization is distributed across numerous resources - documentation, business process models, software components and artifacts. However not only organizational internal resources possess vital knowledge for business process analysis - external repositories of legal documents, regulations and standards hold important information related to business processes as well.

During "as-is" business process modeling, a business process modeler must be presented with existing process knowledge and related information to make an accurate model providing realistic representation of the modeled process. It is necessary to analyze the content and structure of all mentioned sources to extract business process related knowledge and also merge extracted process knowledge.

The author came out with a notion of "business process

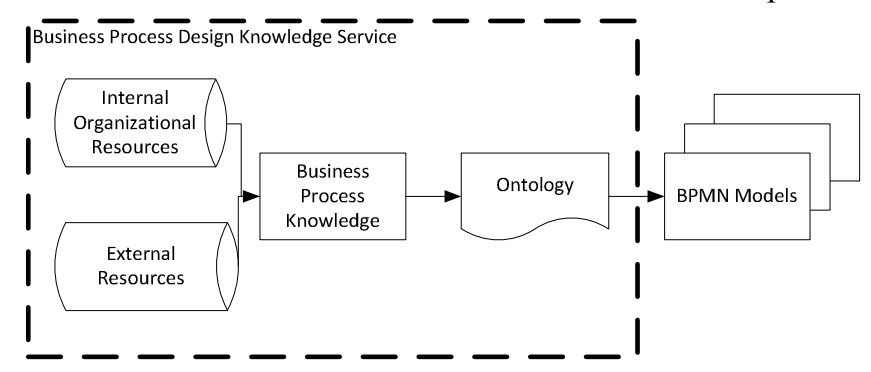

Figure 1. The high-level architecture of business process design knowledge service

knowledge as a service" model in which existing business process knowledge is identified and harvested from organizational internal as well as external resources, integrated and presented to a business process analyst in a comprehensible way. Using extracted business process knowledge a business process analyst can make assessments about existing process peculiarities that might be skipped during documentation analysis, software review and interviews with subject-matter experts. Extracted business process knowledge not only contribute to "as-is" business process analysis and modeling but also generate an input for creating new innovative business processes as described by Bernstein et $a l$. in [4] and creating new service compositions.

The classification of resources storing business process knowledge can be described as follows:

- Internal organizational resources - documentation and software possessed by organization, e.g. legacy systems [5], web applications [6], workflow systems execution logs [7]. However not always organization will have access to source code [6].

- External resources - legislation, regulations, process frameworks and standards are stored, defined and maintained externally to the business process definition and are independent of it, also they may be reusable and applicable across many business processes [8].

According to Xiao et al. [9] business process specification

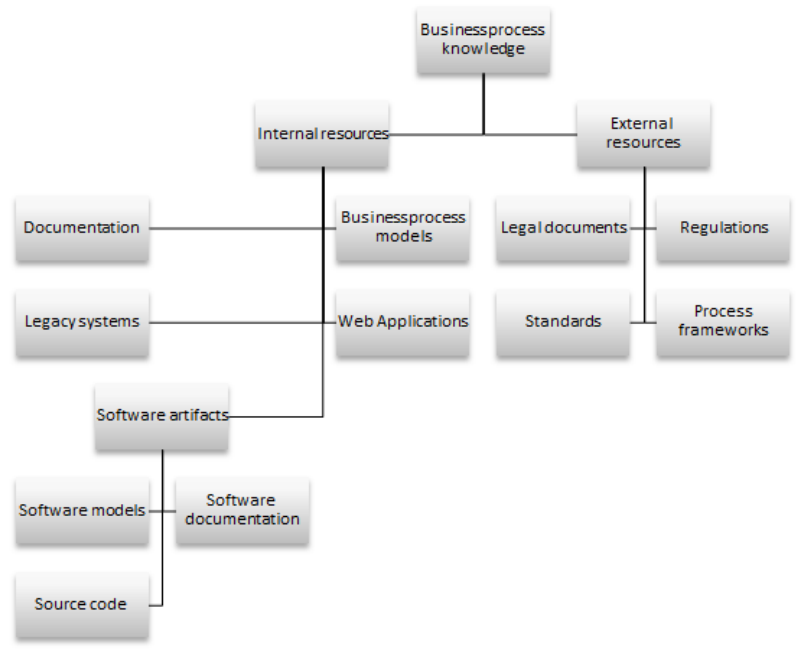

Figure 2. Classification of resources storing business process knowledge

languages, such as BPMN [2] and BPEL [10], are designed to describe executable or abstract business processes and are not suitable for representing the extracted process knowledge. Some BPM platforms (e.g. ARIS, IBM WebSphere) provide tools for identifying new knowledge from business process models. This project will create add-on service for deriving and passing existing knowledge from external source to business process design tool. Kim et al. [11] identify difficulty in querying and reusing business processes as one of the major problems preventing Business Process Management from successful implementation.

\section{PROPOSED METHODOLOGY}

The proposed methodology for this research is a design science method using deductive research approach. Based on this methodology the activities of service design for business process knowledge will be accomplished. 


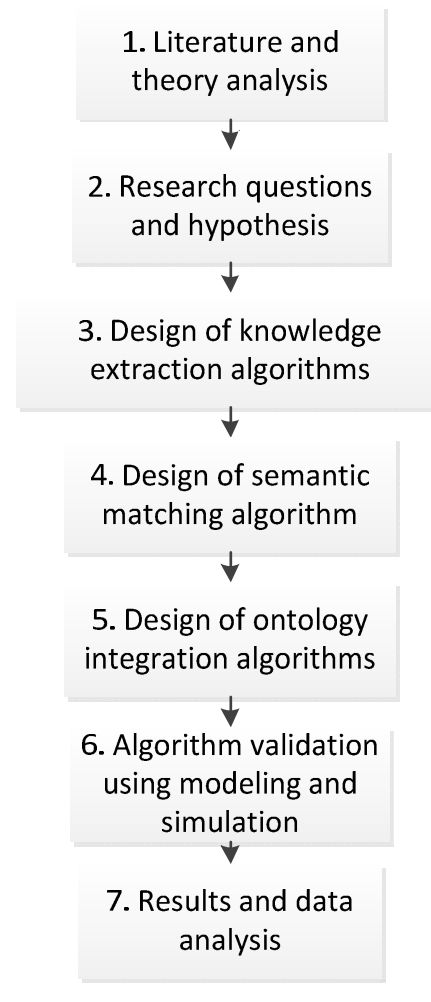

Figure 3. The proposed methodology

The validation of the algorithms implemented in the business process knowledge service will be conducted using modeling and simulation. The simulation method will allow to evaluate the service by executing it using a model of the real "as-is" business process model.

\section{PROGRESS AND RESULTS}

The previous research of the author focused on obtaining a formalized description of a business process from BPMN models represented in different dimensions [12]. Obtained results potentially can be used in translating BPMN models to business process ontology in order to extract process knowledge embedded in existing BPMN process models.

At the moment of writing the research progress mostly refers to defining a framework for linking the excerptions of legal documents from an external registry within the business process model (i.e. linking an activity to a specific part or a section of law). The framework also consists of a web-service to monitor the changes made to legal documents or process itself in order to mark the affected process nodes and inform the business process analyst to consider the introduced changes and their impact on business process flow and conformance with new legal requirements.

The defined framework consists of the following:

- Business analyst creates a BPMN business process model and assigns the process elements with special legal requirements URI attributes. These URI localize and are associated with specific excerptions from legal documents.
- Web-service constantly monitors whether changes are made to legal documents and in a case of such changes marks the affected business process nodes and sends notification to a business process analyst, so the business process analyst can update business process models accordingly to new legal requirements.

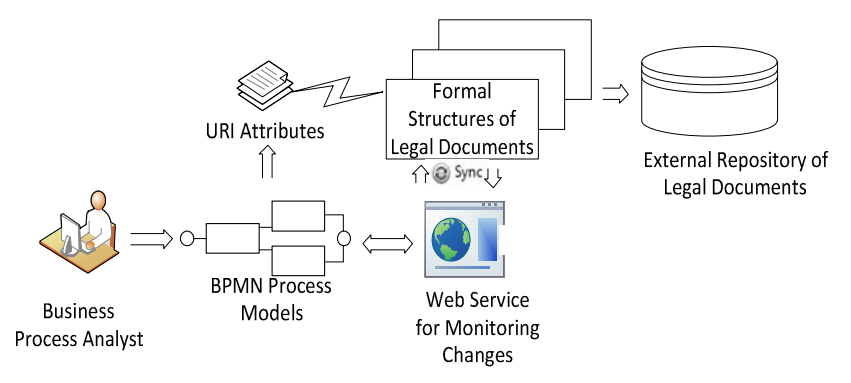

Figure 4. The framework for linking the excerptions of legal documents from an external registry within the business process model

\section{A. $\quad$ Outstanding Issues}

As a result of literature analysis the research challenges and engineering challenges listed below were identified:

- $\quad$ Research challenge of designing business process knowledge extraction algorithms and semantic matching - algorithms for identifying the most relevant business process knowledge and related semantic information for business process modeling in internal and external resources, retrieving identified knowledge and storing it as ontology. The issue of extracting knowledge from existing BPMN models and generating business process ontology from BPMN models must be addressed.

- Research challenge of selection the information retrieval semantic matching metrics - creating a formula for semantic similarity and indicators to identify the relevance of retrieved information and the extent of compliance with the initial request. Aversano et al. [13] apply two widely accepted information retrieval metrics - recall and precision. Xiao et al. [9] propose a metric called average semantic similarity degree to measure the semantic similarity between initial user's request and a website menu.

- Engineering challenge of integration the extracted knowledge in the form of ontologies retrieved from different sources described in Figure 2. Extracted knowledge from different resources must be integrated to form a comprehensive ontology. Xiao et al. [9] propose an algorithm that integrates knowledge extracted from multiple websites.

In order to implement the shown in the Figure 4 legal documents modeling scenarios in organization's everyday business process modeling, it is necessary to introduce such novelties in legal requirements modeling as a whole: 
- Formalizing a structure of legal documents - today mostly the codification of laws and regulation is done with the use of natural language [14]. Formalization of the structure of legal documents will present information in a machine readable format. This will provide a possibility to map a particular part of a legal document to a particular element of a business process model and to enable monitoring of changes obtained to this particular law excerption.

- $\quad$ Supplementing BPMN meta-model with a subprocess type called "Legal Activity", similarly to the existing BPMN “Call Activity" element. This subprocess type will serve as a container for a business process flow with some specific and externally defined legal requirements. "Legal Activity" could be reusable across many business processes. Particular parts of a business process that must be compliant with a law or standard should be modeled as a subprocess, enabling reusability of this subprocess and linking a legal document or regulation to a process flow, not only one element.

- URI attribute should be added to BPMN flow elements in order to define the link between business process node and externally stored XML schemas of legal documents and regulations. The business process analyst should be able to copy the URI of the particular law excerption or law itself and associate it with a particular business process element or set of elements using URI attribute (e.g. by simply copying the required URI from external location).

- To implement synchronization and monitoring of the changes made to the externally stored legal documents that are linked to a business process, a web-service must be developed which continuously checks and harvests for changes and updates introduced to legal documents or business process itself and in case of modifications visualizes the affected process nodes and notifies the business process analyst.

Using web-services to monitor the connected business process nodes with particular excerptions from legal documents that are located externally and independently of a business process model and to synchronize the updates to made to business process and legal documents will make sure the business process uses the latest definition of a legal document and not the definition at the time the business process model was developed.

\section{RELATED WORK}

The issue of retrieving and recovering business process related information has been addressed in numerous studies of process mining and information extraction research areas.

For machine-readable knowledge retrieval, representation and sharing ontologies are widely used. Ontologies use a formal way to represent knowledge as a set of concepts and relationships among the concepts [9]. Papavassiliou et al. [15] define ontology creation and refinement as separate phases in the business process knowledge method to support Business
Process Oriented Knowledge Management (BPOKM). Kim et al. [11] propose defining ontologies associated with business processes on the basis of a formal methodology for ontology development i.e. METHONDOLOGY. According to [11] METHONDOLOGY provides a method for the reuse of the ontology and even allows for its combination and integration with other ontologies.

There exist special tools related to information extraction, e.g. Text2Onto [16] that can extract ontologies from unstructured or semi-structured textual resources [9]. TXL language [17] for identifying and annotating business rules in the source code.

Business process is a sequence of related activities to achieve a particular business goal. Business process activities are composed of different tasks that can be manual, automatic or semi-automatic. Another related research area is a methodology of Task Knowledge Structures (TKS) by Johnsson et al. [18]. TKS represent the different aspects of knowledge that are possessed by a task performer [18] and were originally developed as a means of eliciting and representing task knowledge for the purpose of designing systems and user interfaces to best support task performance [19]. In the context of this research TKS potentially can be applied as an approach for business process task knowledge modeling and as a basic structure for retrieving business process task related knowledge.

In Massachusetts Institute of Technology (MIT) The Process Handbook [20] has been developed in order to create a repository and associated tools to help users effectively retrieve and exploit the process knowledge relevant to their current challenges [4].

Bernstein et al. [4] propose a method and a tool as an addon to the MIT Process Handbook called The Process Recombinator for generating innovative processes from existing process knowledge. The main idea of The Process Recombinator is to retrieve existing process elements and combine them in alternative ways with intension of creating innovative process flow.

Several studies address business process extraction from implemented software and software artifacts. Kalsing et al. [5] proposes an incremental approach for business process and business rules extraction from large legacy systems using process mining technique and IncrementalMiner algorithm for business structure extraction from execution logs. Agrawal et al. [7] present an approach for creating business process models from workflow system execution logs by constructing a graph. Aversano et al. [13] propose a method based on information extraction techniques for recovering traceability links between business process and software system components in two phases - semantic processing and matching processing. Xiao et al. [9] use ontologies to represent the extracted process knowledge. Based on [9] approach this research will apply ontologies in order to generate a formalized structure of the extracted business process knowledge.

Another related field is legal informatics - one of the main issues legal informatics domain addresses is how to link business process models to the legal documents in order to 
create traceable laws models [14]. Also authors of [14] state that legal documents must have a formal, machine-readable structure in order to access legal documents and bond them or specific parts of them to business process model elements as well as process sequence and data flow.

The Legal Knowledge Interchange Format (LKIF) is a semantic web based language for representing legal knowledge in order to support modeling of legal domains and to facilitate interchange between legal knowledge-based systems [21]. Authors of [14] developed OWL-DL ontology in order to add semantic information about processes described in legal texts LKIF core concepts extended with a business process metamodel to borrow several entities from BPMN meta-model.

AKOMA NTOSO schema is a set of XML machinereadable descriptions of official documents such as legislation, debate record, minutes, etc. that allow exchanging and reusing parliamentary, legislative and judiciary documents more efficiently enabling addition of descriptive structure (markup) to the content of parliamentary and legislative documents [22]. By using AKOMA NTOSO schema any part of the XML description of a legal document contains the relevant portion of the legal document itself i.e. the portion of the legal document that the XML element marks up.

Another example of utilizing XML schema for documenting business rules and regulations was created by Object Management Group (OMG) [23] and is called the Semantics of Business Vocabulary and Business Rules Specification (SBVR) [14]. The SBVR specification defines the vocabulary and rules for documenting the semantics of business vocabulary, business facts, and business rules; as well as an XMI schema for the interchange of business vocabularies and business rules among organizations and between software tools [24].

Following the rapid development of the business rules management BPMN 2.0 version provides three constructs related to business rules modeling within a business process [8]:

- Business rule task - special type of task that evaluates a business rule at a particular point in the process. This type of task provides the input data needed to evaluate the rule and receives the result in an output variable.

- Conditional event - is used when BRE continuously monitors some Boolean data condition and publishes the event when the condition becomes true.

- Data object - contains the input data passed from business process model to business rules engine to be evaluated against a ruleset and to send the output data (result) back to the business process. Conditional on the rule result value any action required is modeled as a business process activity.

- Call activity - externally defined subprocess. A call activity represents invocation of either the reusable global task of process. Data must be explicitly passed from the Call Activity to the global task or called process.

\section{CONCLUSION}

The main purpose of this research project is to design a service model for identifying and extracting existing business process knowledge from organizational internal and external sources in order to present integrated business process knowledge to the business process modeler during "as-is" process modeling. Accurate "as-is" business processes cannot be designed only using information gained through interviews with experts, business process related information stored in organizational information systems and external repositories must be taken into account. The service will be potentially useful for organizations in order to conduct qualitative "as-is" process analysis and make decisions considering new process development.

\section{REFERENCES}

[1] J. Jeston, J. Nelis, "Business Process Management", CA: Elsevier, 2008, pp 469.

[2] Object Management Group, "Business Process Model and Notation", Internet: www.bpmn.org, accessed on Feb. 3, 2012.

[3] M. Chinosi, A. Trombetta, "BPMN: An introduction to the standard", in Computer Standards \& Interfaces 34, Elsevier, 2012, pp 124-134.

[4] A. Bernstein, M. Klein, T. Malone, T. W. Malone, "The Process Recombinator : A Tool for Generating New Business Process Ideas" in Proceedings of the International Conference on Information Systems, 1999, pp 178-192.

[5] A. C. Kalsing, G. S. D. Nascimento, C. Iochpe, L. H. Thom, "An Incremental Process Mining Approach to Extract Knowledge from Legacy Systems", 14th IEEE International Enterprise Distributed Object Computing Conference, 2010, pp 79-88, Ieee. doi:10.1109/EDOC.2010.13.

[6] C. Di Francescomarino, A. Marchetto, P. Tonella, "Reverse Engineering of Business Processes exposed as Web Applications", 2009, 13th European Conference on Software Maintenance and Reengineering, pp 139-148. Ieee. doi:10.1109/CSMR.2009.26.

[7] R. Agrawal, D. Gunopulos, F. Leymann, "Mining Process Models from Workflow Logs", in Lecture Notes in Computer Science: Advances in Database Technology — EDBT'98, vol. 1377/1998, 1998, pp. 469-483, Springer Berlin / Heidelberg. doi:10.1007/BFb0101003.

[8] B. Silver, "BPMN Method and Style", CA:Cody-Cassidy Press, 2009, pp 213.

[9] H. Xiao, J. Ng, A. Lau, "An Automatic Approach for Extracting Process Knowledge from the Web", IEEE International Conference on Web Services (ICWS), 2011, pp.315-322, doi: 10.1109/ICWS.2011.85.

[10] Web Services Business Process Execution Language Version 2.0, http://docs.oasis-open.org/wsbpel/2.0/OS/wsbpel-v2.0-OS.html, accessed on Feb. 3, 2012.

[11] G. Kim, "Ontology-based Semantic Matching for Business Process Management", in Data Base For Advances In Information Systems, vol. 41(4), 2011, pp 98-118.

[12] L. Penicina, "Towards the Mapping of Multidimensional BPMN Models to Process Definition Standards" in Applied Computer Science, Scientific Journal of RTU, vol. 43, 2010, pp 76-83.

[13] L. Aversano, F. Marulli, M. Tortorella, "Recovering Traceability Links between Business Process and Software System Components" in IEEE 18th International Conference on Program Comprehension, 2010, pp 5253, Ieee. doi:10.1109/ICPC.2010.54.

[14] A. Ciaghi, K. Weldemariam, A. Villafiorita "Law Modeling with Ontological Support and BPMN: a Case Study" in CYBERLAWS 2011, The Second International Conference on Technical and Legal Aspects of the e-Society, IARIA, 2011. 
[15] G. Papavassiliou, S. Ntioudis, G. Mentzas, A. Abecker, "Business process knowledge modelling: method and tool", in Database and Expert Systems Applications, 2002. Proceedings. 13th International Workshop 2010, pp. 138-142.

[16] Text2Onto http://code.google.com/p/text2onto/, accessed on Feb. 3, 2012.

[17] Txl http://www.txl.ca/, accessed on Feb. 3, 2012.

[18] H. Johnson, P. Johnson, „Task Knowledge Structures: Psychological basis and integration into system design", in Acta Psychologica, 78, 1991 pp 3-26.

[19] K. J. Fadel, S. A. Brown, “A Framework for Knowledge Transfer in Process Redesign” in SIGMIS Database, 2008, vol. 39(3), pp 21-40.
[20] The MIT Process Handbook Project http://ccs.mit.edu/ph/, accessed on Feb. 3, 2012.

[21] Legal Knowledge Interchange Format, http://www.estrellaproject.org, accessed on Feb. 3, 2012.

[22] Akoma NTOSO, http://www.akomantoso.org/, accessed on Feb. 3, 2012.

[23] Object Management Group, http://www.omg.org/, accessed on Feb. 3, 2012.

[24] Semantics of Business Vocabulary and Business Rules (SBVR), Version 1.0, http://www.omg.org/spec/SBVR/1.0/, accessed on Feb. 3, 2012 